PROCEEDINGS OF THE

AMERICAN MATHEMATICAL SOCIETY

Volume 125, Number 1, January 1997, Pages 17-26

S 0002-9939(97)03930-0

\title{
ON 2D PACKINGS OF CUBES IN THE TORUS
}

\author{
ANDREW V. REZTSOV AND IAN H. SLOAN \\ (Communicated by William W. Adams)
}

\begin{abstract}
The 2D packings of cubes (i.e. squares) in the torus $\mathcal{T}^{2}=[0,1)^{2}$ are considered. We obtain the exact expression $N_{2}(\lambda)=\lfloor\lambda\lfloor\lambda\rfloor\rfloor$ for the quantity $N_{2}(\lambda)$, the maximal number of $2 \mathrm{D}$ cubes in a packing. (Here $1 / \lambda$ is the length of sides of cubes, $\lambda \in \mathbf{R}, \lambda>2$.) Corresponding best packings are constructed. Both rank 1 best lattice packings and rank 2 best lattice packings are given.
\end{abstract}

\section{INTRODUCTION}

This article is concerned with the classification and investigation of the $2 D$ best packing of cubes (i.e. squares) in the standard torus $\mathcal{T}^{2}=[0,1)^{2}$.

First of all we consider lattice packings; some of these will turn out to be extremal for the packing problem. According to the classification in [1] or [2], the lattices (in our 2D case) are either rank 1 lattices or rank 2 lattices. First, we study lattices of the rank 1 form,

$$
\left(\left\{\frac{k a_{1}}{N}\right\},\left\{\frac{k a_{2}}{N}\right\}\right) \in \mathcal{T}^{2}, \quad k=0, \cdots, N-1,
$$

where $1 \leq a_{1}<a_{2}<N, a_{j} \in \mathbf{N}(j=1,2)$. We denote by $\{t\}$ the fractional part of the number $t$. The lattice (1) is of rank 1 , because it consists of integer multiples (modulo 1) of the single vector $\left(a_{1}, a_{2}\right) / N$.

We also consider rank 2 lattices. For example, if $n \geq 2$ the lattice

$$
\left(\frac{k_{1}}{n}, \frac{k_{2}}{n}\right) \in \mathcal{T}^{2}, \quad k_{1}, k_{2}=0, \cdots, n-1,
$$

is of rank 2 (the maximum for a $2 \mathrm{D}$ lattice). We shall be interested in rank 2 lattices of the more general form [1]:

$$
\begin{gathered}
\left(\left\{\frac{j a_{1}}{n r}+\frac{k_{1}}{n}\right\},\left\{\frac{j a_{2}}{n r}+\frac{k_{2}}{n}\right\}\right) \in \mathcal{T}^{2}, \\
k_{1}, k_{2}=0, \cdots, n-1, j=0, \cdots, r-1 .
\end{gathered}
$$

Later we shall use (1)-(3) as a foundation for studying best packing.

Received by the editors June 16, 1995.

1991 Mathematics Subject Classification. Primary 05B40; Secondary 11H31, 52C15, 65D32. 
In conformity with the traditional packing problem, for $1 \leq p \leq \infty$ we define spheres

$$
B_{p}\left(x^{(0)} ; R\right)=B_{p}^{s}\left(x^{(0)} ; R\right)=\left\{x \in l_{p}^{(s)}:\left|x-x^{(0)}\right| \leq R\right\} .
$$

Here $s$ is the dimension, $x^{(0)}$ the sphere center and $R$ the sphere radius; and $l_{p}^{(s)}$ is the space with the metric

$$
|x-y|=\left(\sum_{j=1}^{s}\left|x_{j}-y_{j}\right|^{p}\right)^{1 / p},
$$

where $x=\left(x_{1}, \cdots, x_{s}\right), y=\left(y_{1}, \cdots, y_{s}\right) \in \mathbf{R}^{s}$. In particular, when $p=\infty$ and $s=$ 2 the spheres become squares.

Definition 1.1. A packing of spheres $B_{p}^{s}(\cdot ; R)$ in an $s$-dimensional manifold $M$ is a system

$$
\left\{B_{p}^{s}\left(x^{(k)} ; R\right)\right\}_{k=1}^{N}
$$

where

$$
\begin{gathered}
B_{p}^{s}\left(x^{(k)} ; R\right) \subset M, \quad k=1, \cdots, N, \\
\operatorname{mes}_{\mathbf{R}^{s}}\left\{B_{p}^{s}\left(x^{\left(k_{1}\right)} ; R\right) \cap B_{p}^{s}\left(x^{\left(k_{2}\right)} ; R\right)\right\} \\
=0, \\
k_{1} \neq k_{2}, k_{1}, k_{2}=1, \cdots, N .
\end{gathered}
$$

We take in our case $s=2, p=\infty, M=\mathcal{T}^{2}$, and study the quantity $N_{2}(\lambda)$, which is the maximal number of spheres (i.e. squares) with sides of length $1 / \lambda$ in the packing. Such packings are of use for some number theory problems [3], [4], although generally speaking the interesting question is the behavior of $N_{s}(\lambda)$ for all $s \in \mathbf{N}, s \geq 2$.

The general problem of determining $N_{s}(\lambda)_{p}(1 \leq p \leq \infty)$ is not solved yet. V. A. Yudin [5] presented a promising approach: a duality between some extremal problems for trigonometric polynomials and the packing problem in Euclidean space. We face the problem of the asymptotic behavior of the packing fraction

$$
\frac{N_{s}(\lambda)_{p}}{\lambda^{s}}
$$

when $s \rightarrow \infty$. Even the existence of a limit of the packing fraction is not proved so far. However, for $p=\infty$ packings and the packing fraction were studied by S. B. Stechkin [3], who showed:

For arbitrary fixed $\lambda>2$ there exists a number $A=A(\lambda)$

$$
\text { such that } \frac{1}{s} \ln N_{s}(\lambda)=A\left(1+o\left(s^{-1}\right)\right) \quad(s \rightarrow \infty) .
$$

We denote by $\lfloor t\rfloor$ the integer part of the number $t$. The main result of this paper is the following explicit expression for $N_{2}(\lambda)$. The proof is carried out through the construction of explicit lattices for which $N=N_{2}(\lambda)$, thus best packings are constructed for all $\lambda>2$. 
Theorem 1.1.

$$
N_{2}(\lambda)=\lfloor\lambda\lfloor\lambda\rfloor\rfloor .
$$

The result is of course trivial if $\lambda$ is an integer.

\section{THE UPPER BOUND}

We first establish the upper bound

$$
N_{2}(\lambda) \leq\lfloor\lambda\lfloor\lambda\rfloor\rfloor,
$$

giving an explicit proof as a matter of convenience; the method used in this proof is of common knowledge and gives the more general bound

$$
N_{s}(\lambda) \leq\left\lfloor\lambda \cdot N_{s-1}(\lambda)\right\rfloor \leq\lfloor\lambda\lfloor\lambda\lfloor\lambda \cdots\rfloor\rfloor\rfloor
$$

in the $s$-dimensional case. Note that there are $s$ pairs of square brackets in (10).

Let $P=P(\lambda)$ be an arbitrary best packing of squares, with $1 / \lambda$ the length of sides of the squares (i.e. the radii of the spheres equal $1 /(2 \lambda)$ ):

$$
P=\left\{B_{\infty}^{2}\left(x^{(k)} ; 1 /(2 \lambda)\right)\right\}_{k=1}^{N_{2}(\lambda)} .
$$

Denote by $K=K(P)$ the set

$$
K=\left\{x \in \mathcal{T}: x \notin B_{\infty}^{2}\left(x^{(k)} ; 1 /(2 \lambda)\right) \in P, \quad k=1, \cdots, N_{2}(\lambda)\right\} .
$$

Consider the function

$$
f_{P}(x)= \begin{cases}\lambda^{2}, & \text { if } x \in \mathcal{T} \backslash K, \\ 0, & \text { otherwise }\end{cases}
$$

One can see that

$$
N_{2}(\lambda)=\int_{0}^{1} \int_{0}^{1} f_{P}(x) d x .
$$

After the reduction of the double integral (11) to an iterated integral we obtain the inequality

$$
N_{2}(\lambda) \leq\left(\int_{0}^{1 / \lambda} \lambda^{2} d x\right) \cdot N_{1}(\lambda) .
$$

Because $N_{1}(\lambda)=\lfloor\lambda\rfloor$ and $N_{2}(\lambda) \in \mathbf{N}$, the estimate (12) implies (9).

\section{THE LOWER BOUND}

Obviously an arbitrary $\lambda \in \mathbf{R}(\lambda \geq 2)$ can be expressed in the form

$$
\lambda=\lfloor\lambda\rfloor+\frac{m}{\lfloor\lambda\rfloor}+\frac{\varepsilon}{\lfloor\lambda\rfloor},
$$

where $m=0, \cdots,\lfloor\lambda\rfloor-1,0 \leq \varepsilon<1$. Consider the rank 1 lattice (1) with

$$
a_{1}=1, a_{2}=\lfloor\lambda\rfloor, \quad N=\lfloor\lambda\rfloor^{2}+m=\lfloor\lambda\lfloor\lambda\rfloor\rfloor .
$$

Now we have to check whether this lattice can be the set of centers of the packing with the side length of squares equal to $1 / \lambda$. So the fulfillment of the condition (7) with $R=\lambda / 2$ needs to be tested. 
For the case of a lattice it is obvious that one center of the two squares in (7) can be arbitrary and fixed, thus we assume $k_{1}=0$. Furthermore, we may restrict the range of $k_{2}$,

$$
k_{2}=1, \cdots,\left\lfloor\frac{N}{2}\right\rfloor \text {. }
$$

First assume that $\lfloor\lambda\rfloor \leq k_{2} \leq\left\lfloor\frac{N}{2}\right\rfloor$. Then the first coordinate of $x^{\left(k_{2}\right)}$ satisfies

$$
\left\{\frac{k_{2}}{N}\right\}=\frac{k_{2}}{N} \leq \frac{1}{2} \text {. }
$$

Denote by $(x)_{l_{\infty}^{(2)}}$ the distance (in the space $l_{\infty}^{(2)}$ ) between $x \in l_{\infty}^{(2)}$ and the nearest integer vector $z \in \mathbf{Z}^{2}$. Then by (15) we see that

$$
\left(x^{\left(k_{2}\right)}\right)_{l_{\infty}^{(2)}} \geq \frac{k_{2}}{N} \geq \frac{\lfloor\lambda\rfloor}{N} \geq \frac{1}{\lambda}, \quad\lfloor\lambda\rfloor \leq k_{2} \leq\left\lfloor\frac{N}{2}\right\rfloor .
$$

Now consider the case $1 \leq k_{2} \leq\lfloor\lambda\rfloor-1$. Then the second coordinate of $x^{\left(k_{2}\right)}$ satisfies

$$
\left\{\frac{k_{2} \cdot\lfloor\lambda\rfloor}{N}\right\}=\frac{k_{2} \cdot\lfloor\lambda\rfloor}{N} \geq \frac{\lfloor\lambda\rfloor}{N} \geq \frac{1}{\lambda}
$$

and

$$
\left\{\frac{k_{2} \cdot\lfloor\lambda\rfloor}{N}\right\} \leq \frac{(\lfloor\lambda\rfloor-1)\lfloor\lambda\rfloor}{N} \leq 1-\frac{1}{\lambda} .
$$

Comparing (17) and (18) gives

$$
\left(x^{\left(k_{2}\right)}\right)_{l_{\infty}^{(2)}} \geq \frac{1}{\lambda}, \quad k_{2}=1, \cdots,\lfloor\lambda\rfloor-1 .
$$

The correctness of (7) follows from (16) and (19). Thus a packing with $N=$ $\lfloor\lambda\lfloor\lambda\rfloor\rfloor$ squares of side $1 / \lambda$ is constructed (see (14)), and the lower estimate

$$
\lfloor\lambda\lfloor\lambda\rfloor\rfloor \leq N_{2}(\lambda)
$$

is now proved.

Comparing estimates (9) and (20) gives (8).

\section{EXAMPLES AND REMARKS}

Example 4.1. The lattice given by (1), (14) with $\lambda=5 / 2$ (in which as a result $\lfloor\lambda\rfloor=2, m=1, \varepsilon=0$ and $\left.N=N_{2}(5 / 2)=5\right)$ is noteworthy, it being one of the Fibonacci lattices: these are the lattices of the form

$$
\left(\frac{k}{F_{l}},\left\{\frac{k \cdot F_{l-2}}{F_{l}}\right\}\right), \quad k=0, \cdots, F_{l}-1
$$

where $F_{1}=F_{2}=1, F_{l}=F_{l-1}+F_{l-2}$ for $l \geq 3$. Fibonacci lattices (21) are of use as a foundation for cubature formulae, and have been studied extensively [6]-[9]. However, only one Fibonacci lattice, namely that with $l=5$ and $F_{l}=5$, is extremal for the packing problem.

We pay attention to Fibonacci lattices and the corresponding packings because of their interesting geometrical properties. Recently H. Niederreiter and I.H. Sloan proved the following result [8]. 
Theorem A. For $l=2 t+1$ with $t=1,2, \ldots$ the Fibonacci lattice (21) has a square unit cell determined by the vectors

$$
\begin{aligned}
& \mathbf{a}=\left(F_{t} / F_{2 t+1},(-1)^{t-1} F_{t+1} / F_{2 t+1}\right) \text { and } \\
& \mathbf{b}=\left(F_{t+1} / F_{2 t+1},(-1)^{t} F_{t} / F_{2 t+1}\right) .
\end{aligned}
$$

For the case $l=2 t+1$ we can therefore calculate the maximal length $l_{\max }(t)$ of packing squares in the Fibonacci lattice: since the distance (in the maximum norm) of the nearest non-lattice points is $F_{t+1} / F_{2 t+1}$, the maximal length is

$$
l_{\text {max }}(t)=\frac{F_{t+1}}{F_{2 t+1}} .
$$

Hence the corresponding extremal value of $\lambda(t)$ is

$$
\lambda(t)=\frac{1}{l_{\max }(t)}=\frac{F_{2 t+1}}{F_{t+1}}
$$

and the number of squares in this packing is $N=F_{2 t+1}$. On the other hand, the corresponding optimal value of $N$ is

$$
N_{2}(\lambda(t))=\lfloor\lambda(t)\lfloor\lambda(t)\rfloor\rfloor=\left\lfloor\left(F_{2 t+1} / F_{t+1}\right)\left\lfloor F_{2 t+1} / F_{t+1}\right\rfloor\right\rfloor .
$$

For instance

$$
\begin{aligned}
& \lambda(1)=2, N_{2}(\lambda(1))=4>F_{2 t+1}=2 ; \\
& \lambda(2)=5 / 2, N_{2}(\lambda(2))=5=F_{2 t+1} ; \\
& \lambda(3)=13 / 3, N_{2}(\lambda(3))=17>F_{2 t+1}=13 ; \\
& \lambda(4)=34 / 5, N_{2}(\lambda(4))=40>F_{2 t+1}=34 ; \\
& \lambda(5)=89 / 8, N_{2}(\lambda(5))=122>F_{2 t+1}=89 ; \\
& \lambda(6)=233 / 13, N_{2}(\lambda(6))=304>F_{2 t+1}=233 ; \ldots
\end{aligned}
$$

To obtain an asymptotic result we can consider the quality deficiency of the Fibonacci rule, defined by

$$
q(t)=\frac{N_{2}(\lambda(t))-F_{2 t+1}}{N_{2}(\lambda(t))}=1-\frac{F_{2 t+1}}{\left\lfloor\left(F_{2 t+1} / F_{t+1}\right)\left\lfloor F_{2 t+1} / F_{t+1}\right\rfloor\right\rfloor} .
$$

For instance

$$
\begin{aligned}
& q(1)=2 / 4=1 / 2 ; \\
& q(2)=0 / 5=0 ; \\
& q(3)=4 / 17 ; \\
& q(4)=6 / 40=3 / 20 ; \\
& q(5)=33 / 122 ; \\
& q(6)=71 / 304 ; \ldots
\end{aligned}
$$

We can use the following property of Fibonacci numbers (see [8, equation (2.2)])

$$
F_{t}^{2}+F_{t+1}^{2}=F_{2 t+1}
$$

together with

$$
\frac{F_{t}}{F_{t+1}} \rightarrow \alpha \quad \text { as } \quad t \rightarrow \infty
$$


where

$$
\alpha=\frac{\sqrt{5}-1}{2}
$$

to obtain

$$
q(t) \rightarrow \frac{\alpha^{2}}{1+\alpha^{2}}=0.276393 \approx 28 \%
$$

Summing up the results, we can say that Fibonacci lattice packing with $l=2 t+1$ is asymptotically worse than the best lattice packing, from the present point of view, by about 28 percent.

\section{Alternative Best PaCKings}

An explicit best packing, namely (14), is given in the proof of Theorem 1.1. In this section we give alternative best lattice packings. One is a family of rank 1 packings generalising (14), the other is a rank 2 packing.

Let the integer $m$ in the representation (13) of $\lambda$ be coprime with $\lfloor\lambda\rfloor$, and let $\epsilon=0$. For any $m_{1}$ such that

$$
m_{1} \mid m
$$

consider the lattice (1) with

$$
a_{1}=m_{1}, a_{2}=\lfloor\lambda\rfloor, \quad N=N_{2}(\lambda)=\lfloor\lambda\rfloor^{2}+m
$$

If $m_{1}=1$ we recover (14). For any value of $m_{1}$ that divides $m$ the rank 1 lattice (1), (22), (23) gives a best packing, as we now prove by showing that (7) is satisfied with $R=1 /(2 \lambda)$ and sphere centres given by

$$
x^{(r)}=\left(\left\{\frac{r m_{1}}{N}\right\},\left\{\frac{r\lfloor\lambda\rfloor}{N}\right\}\right), \quad r=0, \cdots, N-1 .
$$

Because $m$ and $\lfloor\lambda\rfloor$ are coprime it follows that $m_{1}$ and $N=\lfloor\lambda\rfloor^{2}+m$ are coprime too. It follows also that $N$ and $\lfloor\lambda\rfloor$ are coprime. From the first of these, there exist integers $t$ and $s$ such that

$$
m_{1} t+N s=\lfloor\lambda\rfloor .
$$

Now we introduce the number

$$
M=\lfloor\lambda\rfloor t+m_{2},
$$

where

$$
m_{2}=\frac{m}{m_{1}}
$$

and $t$ is as in (24). Next we transform (24) and (25) to the form

$$
\begin{gathered}
m_{1} t+N s-\lfloor\lambda\rfloor=0, \\
\lfloor\lambda\rfloor t-M+m_{2}=0 .
\end{gathered}
$$

Multiplying (27) by $m_{2}$ and (28) by $\lfloor\lambda\rfloor$ and adding gives

$$
\left(m_{1} m_{2}+\lfloor\lambda\rfloor^{2}\right) t+N s m_{2}-M\lfloor\lambda\rfloor=0 .
$$

But (26) is equivalent to

$$
m_{1} m_{2}+\lfloor\lambda\rfloor^{2}=N
$$


which with (29) gives

$$
M\lfloor\lambda\rfloor=N v, \quad \text { for some } v \in \mathbf{Z} .
$$

Because $\lfloor\lambda\rfloor$ and $N$ are coprime, it follows in turn that

$$
M=N w, \quad \text { for some } w \in \mathbf{Z} .
$$

Hence we can write equation (25) in the form

$$
\lfloor\lambda\rfloor t=N w-m_{2} \text {. }
$$

Now let $t_{0}$ (with $0 \leq t_{0} \leq N-1$ ) be the residue of $t$ modulo $N$. Then with the aid of (24) and (31) we obtain the following expression for the point $x^{\left(t_{0}\right)}$ of the lattice (1), (23),

$$
x^{\left(t_{0}\right)}=\left(\left\{\frac{t_{0} m_{1}}{N}\right\},\left\{\frac{t_{0}\lfloor\lambda\rfloor}{N}\right\}\right)=\left(\left\{\frac{t m_{1}}{N}\right\},\left\{\frac{t\lfloor\lambda\rfloor}{N}\right\}\right)=\left(\frac{\lfloor\lambda\rfloor}{N}, 1-\frac{m_{2}}{N}\right) .
$$

We also have

$$
x^{(1)}=\left(\frac{m_{1}}{N}, \frac{\lfloor\lambda\rfloor}{N}\right) .
$$

On noting that

$$
\frac{m_{1}}{N}<\frac{1}{\lambda}=\frac{\lfloor\lambda\rfloor}{N}, \quad \frac{m_{2}}{N}<\frac{1}{\lambda}=\frac{\lfloor\lambda\rfloor}{N},
$$

we see that the four points

$$
x^{(1)}, x^{(N-1)}, x^{\left(t_{0}\right)}, x^{\left(N-t_{0}\right)}
$$

are all at a distance (in the space $l_{\infty}^{(2)}$ and the setting $\mathcal{T}^{2}$ ) of $\lfloor\lambda\rfloor / N=1 / \lambda$ from $x^{(0)}=0$. It only remains to show that no other points of the lattice (1), (23) are closer than $1 / \lambda$ to $x^{(0)}=0$.

To this end we consider the lattice in $\mathbf{R}^{2}$ defined by

$$
\mathcal{L}_{1}=\left\{j_{1} \mathbf{a}+j_{2} \mathbf{b}: j_{1}, j_{2} \in \mathbf{Z}\right\},
$$

where

$$
\begin{gathered}
\mathbf{a}=\left(m_{1} / N,\lfloor\lambda\rfloor / N\right)=x^{(1)}, \\
\mathbf{b}=\left(\lfloor\lambda\rfloor / N,-m_{2} / N\right)=x^{\left(t_{0}\right)}-(0,1) .
\end{gathered}
$$

The volume $V\left(\mathcal{L}_{1}\right)$ of the unit cell of the lattice $\mathcal{L}_{1}$ is

$$
\begin{gathered}
V\left(\mathcal{L}_{1}\right)=\left|\operatorname{det}\left(\begin{array}{ll}
\mathbf{a}_{1} & \mathbf{a}_{2} \\
\mathbf{b}_{1} & \mathbf{b}_{2}
\end{array}\right)\right| \\
=\left|\operatorname{det}\left(\begin{array}{cc}
m_{1} / N & \lfloor\lambda\rfloor / N \\
\lfloor\lambda\rfloor / N & -m_{2} / N
\end{array}\right)\right|=\left|\frac{-m_{1} m_{2}-\lfloor\lambda\rfloor^{2}}{N^{2}}\right|=\frac{1}{N} .
\end{gathered}
$$

The lattice $\mathcal{L}_{1}$ therefore contains exactly $N$ points in $[0,1)^{2}$. If we now identify points of $\mathcal{L}_{1}$ that differ by integer vectors, then it is clear that $\mathcal{L}_{1}$ is a sublattice of $\mathcal{L}$, since the generators $x^{(1)}$ and $x^{(2)}$ of $\mathcal{L}_{1}$ are two of the points of $\mathcal{L}$. Since $\mathcal{L}_{1}$ has the same order $N$ as $\mathcal{L}$, it follows that $\mathcal{L}_{1}$ and $\mathcal{L}$ coincide.

The representation (34) of the lattice $\mathcal{L}$ allows the distances (in $l_{\infty}^{(2)}$ ) of lattice points from $x^{(0)}=0$ to be computed easily. In particular, the point $j_{1} \mathbf{a}+j_{2} \mathbf{b}$ of $\mathcal{L}_{1}$ (in the setting $\mathbf{R}^{2}$ and the space $l_{\infty}^{(2)}$ ) is easily seen to be at a distance from $x^{(0)}$ 


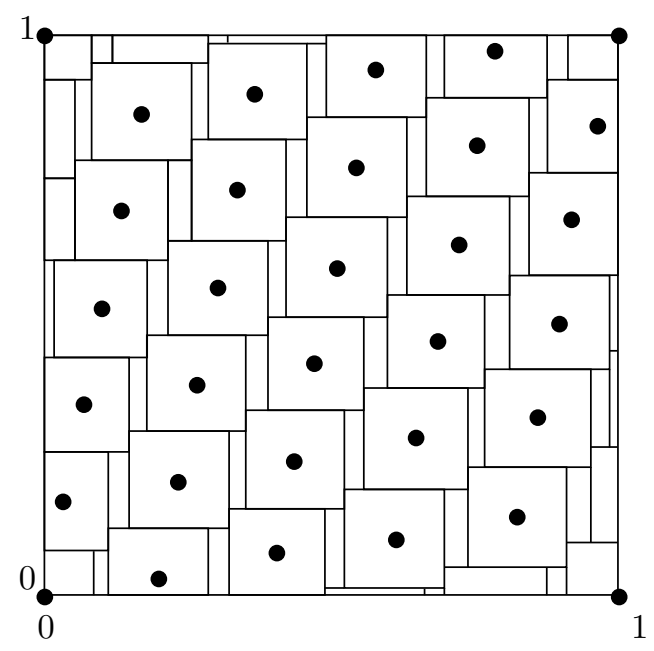

Figure 1

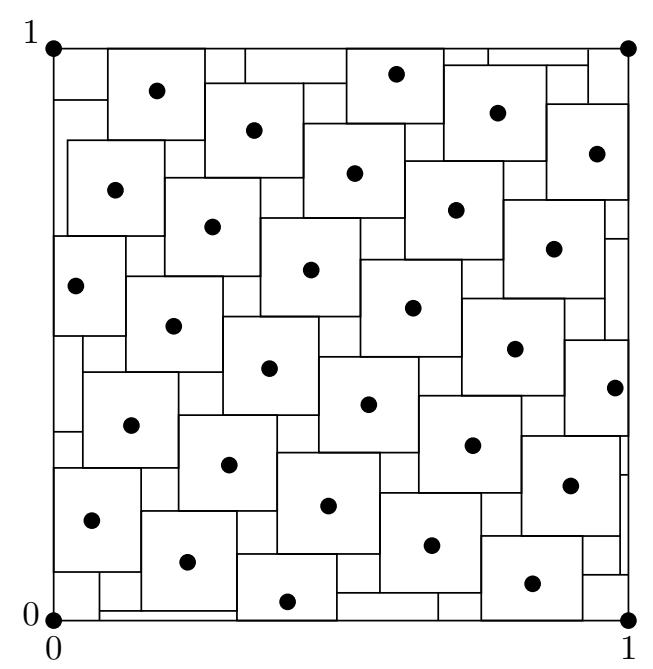

Figure 2

of at least $\left|j_{2}\right|\lfloor\lambda\rfloor / N=\left|j_{2}\right| / \lambda$ if $j_{1}$ and $j_{2}$ are of the same sign or $j_{1}$ is zero; and at least $\left|j_{1}\right|\lfloor\lambda\rfloor / N=\left|j_{1}\right| / \lambda$ if $j_{1}$ and $j_{2}$ are of opposite signs or $j_{2}$ is zero. Thus there are no points closer to $x^{(0)}=0$ than the four points $\mathbf{a},-\mathbf{a}, \mathbf{b},-\mathbf{b}$. These four points correspond to the four points in (33) when lattice points differing by an integer vector are identified.

Consequently, if $m$ is coprime with $\lfloor\lambda\rfloor$, then the rank 1 best packing (14) is not unique. Figures 1 and 2 show the packings (14) and (23) respectively for the case $\lambda=29 / 5,\lfloor\lambda\rfloor=5, m=4$ and $N=29$, with $m_{1}=2$ in the case of Figure 2. For the choice $m_{1}=4$ (not shown) the reader may easily verify that the lattice is just the rotation through a right angle of the lattice in Figure 1. 


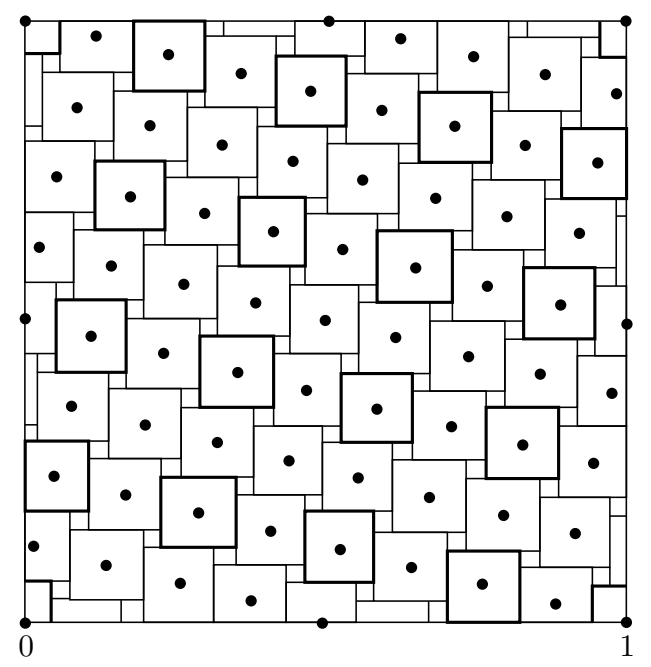

FiguRE 3

Now we construct a rank 2 lattice best packing. Let $\lambda=\lfloor\lambda\rfloor+m /\lfloor\lambda\rfloor$ and $n \in \mathbf{N}(n \geq 2)$ be such that

$$
n m<\lfloor\lambda\rfloor .
$$

Consider the number $\lambda_{*}$ defined by

$$
\lambda_{*}=n \lambda=n\lfloor\lambda\rfloor+\frac{n m}{\lfloor\lambda\rfloor} .
$$

Then (35) and (36) give

$$
\begin{gathered}
\left\lfloor\lambda_{*}\right\rfloor=n\lfloor\lambda\rfloor, \lambda_{*}=\left\lfloor\lambda_{*}\right\rfloor+m_{*} /\left\lfloor\lambda_{*}\right\rfloor, \text { with } m_{*}=n^{2} m, \\
N_{*}=\left\lfloor\lambda_{*}\right\rfloor^{2}+m_{*}=n^{2} N .
\end{gathered}
$$

We assert that the rank 2 lattice of the form (3), where

$$
a_{1}=1, a_{2}=\lfloor\lambda\rfloor, r=N=\lfloor\lambda\rfloor^{2}+m,
$$

is another best packing for squares of side $1 / \lambda_{*}$ and $N_{*}$ points. This can be seen by the following argument. It is easy to see that (3) with $r=N$ is simply the lattice (1) reduced in scale by the factor $n$. (In the language of [1], (39) is an $n^{s}$ copy of the lattice rule (1).) It therefore follows by the second part of the proof of Theorem 1.1 and a trivial geometrical rescaling argument that (7) is satisfied by the rank 2 lattice with parameters (38), and with $R=1 /\left(2 \lambda_{*}\right)=n^{-1} /(2 \lambda)$. On the other hand, the above lattice packing (3), (38) is a best packing, because (using (37))

$$
N_{*}=\left\lfloor\lambda_{*}\left\lfloor\lambda_{*}\right\rfloor\right\rfloor=N_{2}\left(\lambda_{*}\right) .
$$

Figure 3 shows the rank 2 best packing (3), (38) for the particular case

$$
\lambda=17 / 4, \lambda_{*}=17 / 2, n=2, N=17, N_{*}=68 .
$$

This lattice is the $2^{2}$ copy of the 17 point lattice indicated by the highlighted squares in Figure 3. 


\section{ACKNOWLEDGMENT}

The support of the Australian Research Council is gratefully acknowledged.

The research described in this publication was made possible in part by Grants No MC5000 and No MC5300 from the International Science Foundation.

The first author thanks Professor S.B. Stechkin for his help in this research.

\section{REFERENCES}

1. I.H.Sloan and J.N.Lyness, The representation of lattice quadrature rules as multiple sums, Math. Comp. 52 (185) (1989), 81-94. MR 90a:65053

2. I.H.Sloan and S.Joe, Lattice methods for multiple integration, Oxford University Press, 1994.

3. S.B.Stechkin, Some extremal properties of the trigonometric sums, in Modern Problems in Number Theory. Summaries of International Conference, Tula, 1993, p. 153 (Russian).

4. S.B.Stechkin, Some extremal properties of the trigonometric sums, Matematicheskie zametki 55 (2) (1994), 130-143 (Russian). MR 95i:11093

5. V.A.Yudin, Packings of balls in Euclidean space, and extremal problems for trigonometric polynomials, Discretnaya Matematica 1 (2) (1989), 155-158 (Russian); English translation in Discrete. Math. Appl. 1 (1) (1991), 69-72. MR 91a:52023

6. H.Niederreiter, Quasi-Monte Carlo methods and pseudo-random numbers, Bull. Amer. Math. Soc. 84 (1978), 975-1041. MR 80d:65016

7. S.K.Zaremba, Good lattice points, discrepancy and numerical integration, Ann. Mat. Pura Appl. 73 (1966), 293-317. MR 36:1107

8. H.Niederreiter and I.H.Sloan, Integration of nonperiodic functions of two variables by Fibonacci lattice rules, J. Comp. Appl. Math. 51 (1994), 57-70. MR 95f:65056

9. N.S.Bahvalov, Approximate computation of multiple integrals, Vestnik Moskov. Univ. Ser. Mat. Meh. Astr. Fiz. Him. (4) (1959) (Russian). MR 22:6077

10. N.M. Korobov, Number-theoretic methods in approximate analysis, Gosudarstv. Izdat. Fiz.Mat. Lit, Moscow, 1963 (Russian). MR 28:716

Research Fellow, Division of Science and Technology, Tamaki Campus, The UniverSity of Auckland, Private Bag 92019, Auckland, New Zealand

E-mail address: a.reztsov@auckland.ac.nz

School of Mathematics, University of New South Wales, Sydney 2052, New South Wales, Australia

E-mail address: I.Sloan@unsw.edu.au 